\title{
LA ESCALA DE ESTIMA CORPORAL (BES): VALIDACIÓN EN UNA MUESTRA ESPAÑOLA
}

\author{
MERCEDES JORQUERA ${ }^{1}$, ROSA M. BAÑOS ${ }^{1}$, CONXA PERPIÑÁ $^{1}$, CRISTINA BOTELLA $^{2}$ \\ ${ }^{1}$ Universidad de Valencia \\ ${ }^{2}$ Universidad Jaime I de Castellón
}

(Aceptado en febrero de 2005)

\begin{abstract}
Este trabajo tiene como objetivo validar en población española la Escala de Estima Corporal (Body Esteem Scale -BES), elaborada por Franzoi y Shields (1984). La escala fue aplicada a una muestra total de 288 participantes (212 mujeres y 72 varones), con un rango de edad entre 14 y 29 años, procedentes de escuelas, institutos y facultades universitarias. En este estudio se ofrecen datos sobre la consistencia interna, estabilidad temporal (1 mes), validez convergente y análisis factorial. Además, se encuentran diferencias en la estima corporal entre varones y mujeres, en función de la edad, del Índice de Masa Corporal (IMC), y de las puntuaciones en el EAT-40 y la Escala de Restricción.
\end{abstract}

Palabras clave: Imagen corporal, estima corporal, trastornos alimentarios, anorexia nerviosa.

The Body Esteem Scale (BES): Validation in a Spanish sample

The purpose of the present study was to validate in Spanish population the Body Esteem Scale (BES), elaborated by Franzoi \& Shields (1984). A sample of 288 students from primary, secondary and university schools ( 212 women and $72 \mathrm{men}$ ), ranged in age between 14 to 29 years, was administered the BES. In this work, psychometric data are presented, as internal consistency, test-retest reliability ( 1 month), convergent validity and factor analysis. Furthermore, the findings indicate differences in body esteem between men and women, between age groups, BMI (Body Mass Index) groups and between groups according to EAT-40 and Restriction Scale scores

Key words: Body image, body esteem, eating disorders, anorexia nervosa.

\section{INTRODUCCIÓN}

La literatura sobre los trastornos alimentarios sugiere que éstos son de naturaleza multifactorial (p.ej., Fernández y Solé, 2003) y pone de manifiesto que las personas que sufren este tipo de alteraciones y aquellas que están muy preocupadas con su figura, más que tener problemas con su cuerpo, lo que tienen es problemas con cómo representan, evalúan, sienten y viven su cuerpo (Perpiñá, Baños y Botella, 2000). El cuerpo se ha converti-

Correspondencia: Rosa M. Baños, Dpto. Personalidad, Evaluación y Tratamientos Psicológicos, Universidad de Valencia, Av. Blasco Ibáñez, 21, 46010 Valencia. Correo-e: banos@uv.es do en el valor más importante que tienen como personas y es un factor que afecta en gran medida a su autoestima. De ahí, la gran relevancia que tiene, en el ámbito de los trastornos alimentarios, el estudio de la imagen corporal (IC) y, más concretamente, la estima corporal, es decir, cómo las personas valoramos nuestro cuerpo o nuestra apariencia física.

Tradicionalmente, la mayoría de las investigaciones realizadas sobre la IC en personas que padecen de trastornos alimentarios se han dedicado fundamentalmente al estudio de la estimación del tamaño corporal (Raich, 2000). Sin embargo, las investigaciones actuales indican la necesidad de tener en cuenta otros 
aspectos de la IC hasta ahora menos atendidos, como son las emociones, la insatisfacción, las cogniciones, etc. (Raich, 2000). En esta línea, Cash y Deagle (1997) señalan que las medidas actitudinales tienen una capacidad mayor de discriminación entre la población clínica y la no clínica, en comparación con las medidas de la distorsión del tamaño corporal. Sin embargo, contamos con escasos instrumentos para la evaluación de estos componentes; y, más en concreto, son escasos los instrumentos de que disponemos para evaluar la parte afectiva/emocional de la IC; esto es, qué grado de satisfacción producen en la persona cada una de las partes de su cuerpo. No obstante, es necesario no dejar de lado el componente de estima corporal a la hora de evaluar e intervenir sobre las alteraciones de la IC pues, de este modo, abordaremos de manera completa la problemática de este constructo sumamente complejo.

El objetivo fundamental de este trabajo consiste en validar en población española la Escala de Estima Corporal (Body Esteem Scale, BES), elaborada por Franzoi y Shields (1984). Estos autores, desarrollaron este instrumento motivados por las limitaciones con que contaba la Body Cathexis Scale (Secord y Jourard, 1953) que era, hasta ese momento, la única medida que evaluaba la estima corporal, y que contaba con una fiabilidad y validez razonables (Wylie, 1979). Sin embargo, la Body Cathexis Scale adolecía de algunas limitaciones; concretamente, sus autores asumieron que la estima corporal podía expresarse como una única puntuación obtenida a partir del sumatorio de las respuestas que los sujetos daban a los ítems de la escala, esto es, concibieron la estima corporal como un constructo unidimensional. Sin embargo, estudios posteriores realizados a partir de esta escala pusieron en duda el carácter unidimensional del concepto (p.ej., Fisher, 1964; Gunderson y Johnson, 1965), y en algunos trabajos se identificaban diferentes factores en la estima corporal. Por ejemplo, Tucker (1981), tras realizar un análisis factorial sobre la Body Cathexis Scale, identificó los siguientes dominios: salud y estado físico, cara y apariencia global, rasgos corporales subordinados e independientes y fuerza física y muscular. Sin embargo, el tamaño de la muestra de Tucker era muy limitada para aportar datos fiables sobre la estabilidad de los resultados.

Por su parte, Franzoi y Shields (1984) elaboraron un nuevo instrumento, la escala BES, para el cual no asumieron un carácter unidimensional del concepto. La versión final de la escala constó de 35 items, evaluados mediante una escala Likert de 5 puntos donde: 1 significaba "me produce fuertes sentimientos negativos»; 2: «me produce moderados sentimientos negativos»; 3: "no me produce sentimientos ni positivos ni negativos»; 4 : "me produce sentimientos positivos moderados»; y 5: «me produce fuertes sentimientos positivos». La escala fue administrada a una muestra compuesta por 331 hombres y 633 mujeres, todos ellos estudiantes universitarios de Psicología. El análisis factorial de componentes principales obtenido reveló dominios diferentes para los varones y para las mujeres. Para los varones los factores relevantes fueron: "atractivo físico», "resistencia corporal» y «condición física general»; mientras que para las mujeres, los factores relevantes fueron; «atractivo sexual», "preocupación por el peso» y «condición física». En el caso de las mujeres, los factores obtenidos explicaban el $39 \%$ de la varianza, mientras que para los varones la varianza explicada fue de un $35 \%$. En cuanto a la consistencia interna de la escala, se calculó el coeficiente a de Cronbach para cada uno de los factores. Para los varones, se obtuvo un a de .81 para el factor "atractivo físico», de .85 para el de «resistencia corporal» y de .86 para el factor «condición física general». Para las mujeres, los índices de con- 
sistencia interna fueron de .78 para el factor «atractivo sexual»; .87 para el de «preocupación por el peso» y de .82 para el factor «condición física».

Una de las limitaciones del trabajo original, y como reconocen los propios autores, es que la muestra incluida no representaba a todos los grupos de edad. Es más, los autores indican que el empleo de la BES con grupos de edad considerablemente diferentes requeriría desarrollar normas descriptivas adecuadas o, incluso pudiera ser necesario desarrollar de una nueva escala (Franzoi y Shields 1984). En esta misma línea, Mendelson et al. (Mendelson, Mendelson y White, 2001) encontraron que las medidas que Franzoi y Shields describieron en el desarrollo del instrumento, no resultaban adecuadas para jóvenes adolescentes. Por tanto, de los datos expuestos parece desprenderse que la estima corporal es un concepto bastante complejo, que comprende diferentes dominios, los cuales están determinados, en gran medida, por diferentes variables demográficas, entre las que se encuentran el sexo y la edad.

A pesar de las limitaciones señaladas, la BES ha sido la escala más empleada en la evaluación de la estima corporal hasta la actualidad. Diversos estudios avalan sus propiedades psicométricas, indicando que posee una elevada consistencia interna, estabilidad temporal y validez de constructo (Cecil y Stanley, 1997; Franzoi, 1994; Franzoi y Herzog, 1986; Thomas y Freeman, 1990). Sin embargo, se trata de un instrumento únicamente validado con población de habla inglesa. El objetivo fundamental de este trabajo es aportar datos sobre la validación de esta escala en una muestra española. En concreto, los objetivos propuestos son los siguientes: a) traducir la Escala de Estima Corporal (BES) de Franzoi y Shields (1984) a la lengua española; b) comprobar si la estima corporal en una muestra española constituye un constructo unidi- mensional o, por el contrario, está compuesto por diferentes dominios o factores, y si es así, identificarlos; c) analizar si la estima corporal, además del sexo y la edad, depende también de otras variables significativas en el desarrollo de trastornos alimentarios, tales como el índice de masa corporal (IMC), la restricción en la dieta, el esquematismo corporal; d) comprobar la consistencia interna y estabilidad temporal de la escala; y e) comprobar la validez convergente de la escala con otras medidas que también se emplean en la evaluación de la IC en los trastornos alimentarios.

\section{METODO}

\section{Muestra}

La muestra estaba compuesta por 288 participantes reclutados de la población general, de los cuales 212 fueron mujeres (73.6\%) y 72 varones (25\%). El rango de edad de la muestra osciló entre 14 y 29 años, siendo la media para las mujeres de $17,90$ (DT $=3,67)$, y para los hombres de 18,14 (DT = 3,01). En cuanto al índice de masa corporal (IMC), la media para las mujeres fue de 17,9 (DT = 3,67); mientras que para los varones fue de 18,14 (DT $=3,01$ ). La muestra se recabó de centros de EGB, BUP y ESO de las ciudades de Valencia y Alicante, $(\mathrm{N}=147,69,3 \%)$, y de distintas clases de la Universidad Jaime I de Castellón (Facultad de Ciencias Humanas y Sociales). ( $\mathrm{N}=34,16 \%$ ). En el segundo pase de los cuestionarios, un mes después, el $\mathrm{N}$ de la muestra fue de 114 participantes, es decir, el $39,58 \%$ de la muestra original.

\section{Medidas e instrumentos}

Con el fin de obtener información relevante para este estudio, se recogieron 
datos sobre las siguientes variables: a) sexo; b) edad; c) altura; d) peso real (con los datos de altura y peso real se calculaba el IMC); y e) grado de "esquematismo» corporal. Para evaluar la variable «esquematismo", y adaptando la escala que para tal fin crearon originalmente Markus, Hamill y Sentis (1987), se elaboró una escala en que la persona debía describirse según una serie de 6 adjetivos dicotómicos, con un polo positivo y otro negativo, en una escala Likert de 11 puntos. Entre estos adjetivos se encontraban "camuflados» aquellos que hacían referencia al esquematismo para el peso corporal (delgada-gorda). Además, la persona tenía que indicar la importancia que cada una de esas características tenía para ella, según cuatro alternativas de respuesta expresadas en porcentajes $(0 \%$, $25 \%, 75 \%, 100 \%$ ). Posteriormente, gracias a estas puntuaciones se podría diferenciar entre cuatro grupos de participantes. En primer lugar, se diferenció entre "esquemático» $\mathrm{y}$ "no esquemático», en función de si la persona daba mucha importancia al peso corporal (75\% o más) o no ( $25 \%$ o menos). En segundo lugar, a su vez el grupo de esquemáticos se diferenció entre "esquemático-gordo», para aquellos que consideraban que tenían kilos de más; «esquemático-delgado», para quienes pensaban que les faltaba peso; y «esquemático-justo», para aquellas personas que se consideraban en su peso, según el lugar que elegían en la escala bipolar (extremo o central). Originalmente, Markus et al. (1987), plantearon sólo tres grupos (no esquemático, esquemático gordo y esquemático delgado), pero en nuestra investigación se cré un cuarto («esquemático-justo») para poder tener en cuenta a las personas que se consideraban en su peso deseado y eso era muy importante para ellas.

Después de las preguntas que se acaban de mencionar, los participantes completaron una serie de instrumentos que se detallan a continuación (en el orden de aplicación). Todos estos instrumentos, excepto el BSQ y el EAT-40, fueron traducidos por nuestro equipo y la traducción fue revisada por un psicólogo clínico bilingüe. Los instrumentos aplicados fueron los siguientes:

Body Attitude Test (BAT). Test de las Actitudes Corporales (Probst, Vandereycken, Van Coppenolle y Vanderlinden 1995). Evalúa distintos aspectos de la insatisfacción con el cuerpo, como son pensamientos, emociones y las actitudes hacia determinadas características corporales. Esta compuesto por 20 ítems que se evalúan en una escala Likert de 6 puntos (desde nunca hasta siempre). La consistencia interna es de un a de 0,93, y la fiabilidad test-retest es muy alta tanto para pacientes como para población general. El BAT distingue entre pacientes y población general, siendo la puntuación de corte 36, la cual ofrece la mejor proporción entre sensibilidad y especificidad; con un índice de sensibilidad de 0,69 y de especificidad de 0,80 (Probst, et al., 1995). En estudios con población española el coeficiente de consistencia interna es de 0,92, y la fiabilidad test-retest, transcurrida una semana, de 0,91 (Gila, Castro, Gómez, Toro y Salamero, 1999).

Body Esteem Scale (BES). La Escala de Estima Corporal (Franzoi y Shields, 1984). Como ya se ha indicado, esta escala mide la satisfacción/insatisfacción corporal. Consta de 35 items que evalúan el tipo de emociones que le producen a la persona diferentes partes y características corporales, los cuales se valoran en una escala Likert de 5 puntos (1: «fuertes sentimientos negativos»; 2 "sentimientos negativos moderados»; 3 «neutro»; 4 «sentimientos positivos moderados» y 5 «fuertes sentimientos positivos»). Los factores de la escala tanto para varones como para mujeres se han descrito anteriormente. La escala presenta buena fia- 
bilidad en hombres $(0,78-0,87)$ y en mujeres (0,81-0,86) (Thompson, 1995).

Body Image Automatic Thoughts Questionnaire (BIATQ). Cuestionario sobre los Pensamientos Automáticos acerca de la Imagen Corporal (Cash, 1991) Evalúa el componente cognitivo de la IC, concretamente, la frecuencia con la que la persona ha tenido pensamientos negativos o positivos sobre su cuerpo. Está formado por 52 ítems que se dividen en dos subescalas: pensamientos positivos (15 ítems) y pensamientos negativos (37 ítems). Cada pensamiento se puntúa de 1 (nunca) a 5 (muy a menudo). La puntuación total se obtiene restando la puntuación en la escala de pensamientos positivos a la de pensamientos negativos. La escala presenta una consistencia interna de $\mathbf{0 , 9 0}$ para pacientes bulímicas y para población general en ambas subescalas. La fiabilidad test-retest es de 0,90 en la escala negativa y de 0,71 en la escala positiva (citado en Thompson, 1995).

Appearance Schemas Inventory (ASI). Inventario sobre los Esquemas acerca de la Apariencia (Cash y Labarge, 1996). Evalúa el componente cognitivo de la IC: creencias o supuestos específicos sobre la apariencia física y sobre la importancia que ésta tiene en la vida de una persona. Son 14 afirmaciones que se puntúan de 1 (nada de acuerdo) a 5 (totalmente de acuerdo). Las puntuaciones del ASI presentan una consistencia interna de 0,84 y una fiabilidad test-retest de 0,71 .

Situational Inventory of Body-Image Dysphoria (SIBID). «Inventario de malestar provocado por las situaciones relacionadas con la imagen corporal» (Cash, 1994). Recoge las reacciones de malestar e insatisfacción con el cuerpo desencadenadas por determinadas conductas, situaciones o contextos. Incluye 48 situaciones desencadenantes de malestar corporal, como son el ejercicio, el arreglarse, la intimidad, comidas, cambios en el aspecto físico, etc. Evalúa la frecuencia de sentimientos y pensamientos negativos en cada una de esas situaciones en una escala Likert de 5 puntos, en la que 0 es «nunca» y 4 «siempre» (Cash, 1994). A mayor puntuación, la persona presentaría una mayor frecuencia de situaciones en las que experimenta disforia relacionada con la IC. Las puntuaciones del instrumento presentaron una consistencia interna de 0,96 para ambos géneros, y la fiabilidad test-retest ( 1 mes) fue 0,86 para las mujeres y 0,80 para los hombres (Cash, 1994).

Body Areas Satisfaction Scale (BASS). Escala de Satisfacción de Zonas Corporales (Cash, 1991). Evalúa el grado de satisfacción e insatisfacción que una persona tiene respecto a 10 áreas corporales. Se puntúa en una escala tipo Likert que va desde 1 (muy insatisfecho) a 5 (muy satisfecho). Cuenta con una consistencia interna de 0,79 para varones y 0,78 para mujeres (Cash, 1991).

Body Shape Questionnaire (BSQ). Cuestionario de la Forma Corporal (Cooper, Taylor, Cooper y Fairburn, 1986). Está formado por 34 ítems que miden las actitudes de satisfacción / insatisfacción con la figura y preocupación corporal. La escala de respuesta va desde 1 (nunca) hasta 6 (siempre). A mayor puntuación. mayor es la insatisfacción con la imagen corporal. Sirve para discriminar entre personas sin problemas, de las preocupadas por su IC y de las que tienen un trastorno alimentario. Sus coeficientes de consistencia interna $(0,97)$, fiabilidad testretest $(0,88)$, y validez concurrente con otras medidas de la satisfacción corporal son adecuadas (Cooper et al., 1986). En España fue validado por Raich et al. en el año 1996, En España fue validado por Raich. Mora, Soler, Avila, Clos y Zapater (1996). y su puntuación de corte, para población universitaria, es de 105.

Body Image Avoidance Questionnaire (BIAQ; Rosen, Srebrik y Salzberg, 1990). El Cuestionario sobre la Evitación de la Ima- 
gen Corporal evalúa los aspectos conductuales asociados a una imagen corporal negativa. Está compuesto por 19 ítems que recogen los comportamientos llevados a cabo para controlar y disimular la apariencia. El objetivo es evaluar en qué medida las personas llevan a cabo estas conductas de evitación relacionadas con la imagen corporal. Los ítems se responden en una escala de frecuencia de 0 (nunca) a 5 (siempre). A mayor puntuación, más abundantes son las conductas de evitación. El instrumento presenta una consistencia interna de 0,89 y una fiabilidad test-retest a las dos semanas de 0,87 (Rosen et al., 1990).

Eating Attitudes Test (EAT-40). Cuestionario de las actitudes hacia la comida (Garner y Garfinkel, 1979). Está compuesto por 40 ítems que evalúan un amplio rango de actitudes y comportamientos relacionados con la anorexia nerviosa. Cada ítem se puntúa en una escala tipo Likert que va desde nunca hasta siempre. La puntuación total indica gravedad de los síntomas, pero no necesariamente indica un diagnóstico de anorexia nerviosa. El EAT-40 ha mostrado buena consistencia interna $(0,79)$ (Garner y Garfinkel, 1979) y una fiabilidad test-retest satisfactoria a las dos y tres semanas $(0,84)$ (Carter y Moss, 1984). Este instrumento diferencia entre grupos clínicos de aquellos no clínicos y de las personas que padecen un Trastorno por atracón de aquellos que padecen anorexia y bulimia nerviosas. Para este trabajo se empleó la versión y adaptación española realizada por Castro, Toro, Salamero y Guimerá (1991). El análisis de la validez discriminante puso de manifiesto que en la validación española, a diferencia del trabajo original, se recomienda una puntuación de corte de 20 , ya que permite una sensibilidad del $91 \%$ y una especificidad $69,2 \%$.

Restraint Scale (RS). Escala de Restricción (Herman y Polivy,1975). Evalúa las fluctuaciones de peso, el grado de restricción y las actitudes hacia el peso y la comida. Refleja en qué medida una persona posee los patrones de comportamiento alimentario caracterizados por seguir dietas estrictas alternándose con episodios de pérdida de control con la comida. La RS está compuesta por 10 ítems que se responden mediante una escala Likert de 4 ó 5 puntos, según el contenido del item. Consta de dos subescalas: Fluctuación del peso y Preocupación por hacer dieta. Su consistencia interna, para la escala global es mayor de 0,75 y su fiabilidad test-retest es de 0,93 para cuatro semanas y 0,75 para dos años y medio. Según Heatherton, Herman, Polivy, King y McGree (1988) la puntuación de corte es 16.

Rosenberg Self-Esteem Scale. Cuestionario de Autoestima de Rosenberg (RSES; Rosenberg, 1965). Fue diseñado para evaluar los sentimientos globales de autovaloración y auto aceptación. Consta de 10 ítems y, aunque originalmente se elaboró con las características de una escala Guttman, su utilización habitual recurre al formato Likert de cuatro alternativas de respuesta que van desde muy de acuerdo (1) hasta muy en desacuerdo (4). Tiene buenas características psicométricas, ya que su consistencia interna oscilado entre 0,77 y 0,87 (Rosenberg, 1965; Wylie, 1989), y su fiabilidad test-retest es de 0,85 a las dos semanas, y de 0,73 a los siete meses (Wylie, 1989). En población española ha demostrado una consistencia interna de 0,88 (Baños y Guillén, 2000).

\section{Procedimiento}

Los cuestionarios antes mencionados (a excepción de los señalados con un asterisco) fueron traducidos por miembros de nuestro grupo de investigación con un nivel elevado de inglés. Posteriormente, esta traducción fue revisada por un psicólogo clínico bilingüe. El siguiente paso fue solicitar colaboración a diversos centros de enseñanza de la Comunidad Valencia- 
na y desplazamos a esos centros para realizar un pase de test colectivo de una hora de duración. Este mismo procedimiento se siguió al cabo de un mes en el re-test.

\section{RESULTADOS}

\section{Análisis descriptivo de los ítems}

En primer lugar, se realizó un análisis descriptivo global de las puntuaciones dadas a cada uno de los ítems (diferentes partes o aspectos del cuerpo) (ver Tabla 1). En el caso de las mujeres, se observa que, por lo general, la mayoría de las respuestas se sitúan en un rango de neutralidad (en torno a 3). Las partes del cuerpo que generan sentimientos menos positivos hacen referencia a (ver Tabla 1): muslos, peso, caderas y culo (nalgas); mientras que las partes del cuerpo que provocan sentimientos más positivos hacen referencia a: aspecto de los ojos, labios, impulso sexual, salud y reflejos. Es decir, parece que, de manera global, la muestra femenina se siente menos satis-

Tabla 1. Análisis descriptivo de los items del BES

\begin{tabular}{|c|c|c|c|c|}
\hline \multirow[b]{2}{*}{ Ítem del BES } & \multicolumn{2}{|c|}{ Mujeres } & \multicolumn{2}{|c|}{ Varones } \\
\hline & Media & $\mathrm{DT}$ & Media & DT \\
\hline Apetito & 2,94 & 1,08 & 3,76 & 0,97 \\
\hline Nariz & 2,95 & 1,02 & 3,23 & 0,83 \\
\hline Resistencia física & 3,12 & 1,09 & 3,81 & 0,87 \\
\hline Reflejos & 3,59 & 0,88 & 3,88 & 0,82 \\
\hline Labios & 3,78 & 0,93 & 3,69 & 0,90 \\
\hline Fuerza muscular & 3,13 & 0,99 & 3,80 & 0,93 \\
\hline Cintura & 2,99 & 1,13 & 3,37 & 0,87 \\
\hline Nivel de energía & 3,39 & 0,96 & 3,90 & 0,78 \\
\hline Muslos & 2,53 & 1,16 & 3,35 & 1,04 \\
\hline Orejas & 3,26 & 1,07 & 3,17 & 0,88 \\
\hline Bíceps & 2,98 & 0,90 & 3,50 & 0,87 \\
\hline Barbilla & 3,22 & 0,77 & 3,30 & 0,82 \\
\hline Constitución & 3,01 & 1,10 & 3,54 & 0,89 \\
\hline Coordinación física & 3,36 & 0,98 & 3,88 & 0,94 \\
\hline Culo & 2,73 & 1,28 & 3,54 & 0,97 \\
\hline Agilidad & 3,50 & 1,02 & 3,80 & 0,97 \\
\hline Anchura de espaldas & 3,20 & 1,04 & 3,72 & 1,02 \\
\hline Brazos & 3,16 & 0,95 & 3,54 & 0,94 \\
\hline Pechos & 2,95 & 1,14 & 3,24 & 0,91 \\
\hline Aspecto de los ojos & 3,94 & 0,97 & 3,90 & 0,88 \\
\hline Mejilles & 3,47 & 0,89 & 3,36 & 0,70 \\
\hline Caderas & 2,59 & 1,12 & 3,10 & 0,71 \\
\hline Piernas & 2,66 & 1,13 & 3,45 & 0,97 \\
\hline Silueta & 2,92 & 1,13 & 3,38 & 0,85 \\
\hline Genitales & 3,23 & 0.74 & 3,85 & 0,96 \\
\hline Aspecto del estómago & 2,81 & 1,10 & 3,23 & 0,95 \\
\hline Salud & 3,74 & 1,04 & 3,92 & 0,83 \\
\hline Condición física & 3,21 & 0,98 & 3,85 & 0,94 \\
\hline Cara & 3,30 & 1,03 & 3,69 & 0,90 \\
\hline Peso & 2,58 & 1,11 & 3,04 & 1,04 \\
\hline Olor corporal & 2,95 & 5,94 & 2,70 & 1,21 \\
\hline Impulso sexual & 3,72 & 0,83 & 4,32 & 0,80 \\
\hline Pies & 2,91 & 1,06 & 3,14 & 0,86 \\
\hline Vello corporal & 2,64 & 1,10 & 3,04 & 1,06 \\
\hline Actividades sexuales & 3,50 & 1,04 & 3,98 & 0,91 \\
\hline
\end{tabular}


Tabla 2, Resultados del análisis factorial en el grupo de mujeres

\begin{tabular}{|c|c|c|c|}
\hline \multirow[t]{2}{*}{ Item del BES } & \multicolumn{3}{|c|}{ Cargas factoriales } \\
\hline & Preocupación por el peso & Condición física & Atractivo sexual \\
\hline 25. silueta & 0,836 & & \\
\hline 35. peso & 0,776 & & \\
\hline 14. constitución, complexión & 0,767 & & \\
\hline 10. muslos & 0,763 & & \\
\hline 16. culo, nalgas & 0,755 & & \\
\hline 24. piernas & 0,733 & & \\
\hline 23. caderas & 0,705 & & \\
\hline 8. cintura & 0,529 & & \\
\hline 29. aspecto del estómago & 0,451 & & \\
\hline 2. apetito & 0,434 & & \\
\hline 17. agilidad & & 0,744 & \\
\hline 33. condición física & & 0,688 & \\
\hline 15. coordinación física & & 0,685 & \\
\hline 4. resistencia física & & 0,673 & \\
\hline 5. reflejos & & 0,649 & \\
\hline 9. nivel de energía & & 0,581 & \\
\hline 7. fuerza muscular & & 0,577 & \\
\hline 31. actividades sexuales & & 0,448 & \\
\hline 12. bíceps & & 0,424 & \\
\hline 30. salud & & 0,414 & \\
\hline 26. impulso sexual & & 0,413 & \\
\hline 13. barbilla & & & 0,665 \\
\hline 22. mejillas & & & 0,645 \\
\hline 6. labios & & & 0,639 \\
\hline 21. aspecto de los ojos & & & 0,581 \\
\hline 11. orejas & & & 0,568 \\
\hline 30. salud & & & 0,535 \\
\hline 34. cara & & & 0,525 \\
\hline 28. genitales & & & 0,408 \\
\hline
\end{tabular}

Nota. Análisis de componentes principales, rotación varimax.

fecha con las partes del cuerpo relacionadas con el factor «preocupación por el peso", mientras que las variables relacionadas con «atractivo sexual» y «condición física» les producen mayor satisfacción.

En el caso de los varones, por lo general, la media de las puntuaciones suele ser un poco más altas que la de las mujeres, aunque también la mayoría de las respuestas rondan en torno a 3 , es decir, neutralidad. En este caso las puntuaciones más elevadas se dieron a los ítems relacionados con el "atractivo sexual», concretamente, la mayor puntuación la obtuvo el ítem impulso sexual, seguido de actividades sexuales y genitales. Por su parte, las puntuaciones más bajas la obtuvieron ítems correspondientes al factor «apariencia física", siendo los ítems con puntuaciones más bajas: orejas, caderas, peso y vello corporal, correspondiendo este último ítem al factor "fuerza muscular». Parece, por tanto, que los chicos se sienten más satisfechos con las partes del cuerpo relacionadas con el "atractivo sexual», mientras que las variables relacionadas con el factor «apariencia física» y «fuerza muscular" son menos valoradas.

\section{Estructura factorial del BES}

Se realizó un análisis factorial de componentes principales con rotacion vari- 
max con objeto de comprobar si en la población española la estima corporal estaba compuesta por diferentes dominios, tal y como se encontró en el estudio original de Franzoi y Shields. Además, también se pretendía comprobar si se obtenía una estructura similar a la obtenida por los autores de la escala.

Los resultados del análisis de componentes principales para la muestra femenina se presentan en la Tabla 2 . La versión final para nuestra muestra femenina constó de 28 ítems, cada uno de los cuales obtuvo una carga factorial significativa en uno de los tres factores. El punto de corte que se estableció para determinar si un ítem cargaba o no en un factor fue de 0,40 , siendo un poco más estrictos que los autores de la escala original, quienes determinaron como carga significativa los valores iguales o superiores a 0,35 . De los 35 ítems de la escala original se tuvieron que eliminar 7 (Olor corporal; Nariz; Anchura de espaldas; Brazos; Pechos; Pies; Vello corporal) pues ninguno de ellos cargó en alguno de los tres factores. De todos estos ítems eliminados, tres de ellos (Anchura de espaldas; Brazos y Pies) tampoco formaron parte de la estructura que los autores obtuvieron para las mujeres.

Finalmente, la estructura obtenida estuvo compuesta de tres factores, que explican conjuntamente el $46,75 \%$ del total de la varianza. El primero de ellos lo denominamos preocupación por el peso constó de 10 ítems que explicaban el 30,43\% de la varianza. Se mantuvo el mismo nombre que en la escala original puesto que los ítems que lo componen son los mismos que los que componen este factor en la escala original. Las variables que componen este factor hacen referencia a conceptos relacionados con el peso (silueta, constitución, peso, etc.), así como a partes del cuerpo que pueden reflejar de manera más clara el aumento o pérdida de peso (caderas, nalgas, estómago, etc.).
El segundo factor lo denominamos condición física y constó de 11 items (que hacen referencia a la fuerza muscular, energía, grado de salud, resistencia física, etc.), que explicaban el 9,16\% de la varianza $y$, al igual que con el factor anterior, también se conservó el nombre de la escala original, pues también coincidían los items, pero en este caso se añadieron dos más: actividades sexuales e impulso sexual.

Por último, el tercer factor lo denominamos atractivo sexual; compuesto por 8 items (referidos a partes del cuerpo que pueden estar relacionadas con la sexualidad y con la atracción sexual) que explicaban el $7,15 \%$ de la varianza. En este caso, también se conserva el nombre de la escala original, a pesar de que existen diferencias debido a que, por una parte, los ítems que no cargaron en ninguno de los tres factores de nuestra estructura ( $y$ que por tanto fueron eliminados), correspondían a este factor en la escala original; $y$, por otra parte, dos de los ítems que en la estructura original pertenecian a este factor (actividades sexuales e impulso sexual), en nuestra estructura cargaron en el de condición física. Asimismo, en nuestra estructura se añade un ítem más que carga también en el factor de estructura física: salud.

En el caso de los varones, también se realizó un análisis factorial de componentes principales con rotación varimax. Los resultados se presentan en la Tabla 3. También aquí se estableció como punto de corte para determinar si un ítem cargaba o no en un factor la puntuación de 0,40 . La versión final para la muestra masculina constó de 29 items, cada uno de los cuales obtuvo una carga factorial significativa en uno de los tres factores. De los 35 items de la escala original, se eliminaron 6 (Olor corporal; Nariz; Resistencia física; Barbilla; Pechos; Pies) pues ninguno de ellos cargó en alguno de los tres factores. De estos ítems, uno (Olor 
corporal) tampoco formó parte de la estructura que los autores obtuvieron para los varones, sin embargo, otros tres ítems (Piernas; Actividades sexuales; y Vello corporal), que en la escala original no cargaron en ninguno de los factores, en nuestro caso sí que formaron parte de la estructura masculina.

La estructura final que se obtuvo estuvo compuesta de tres factores que conjuntamente explicaban el 47,65\% del total de la varianza. El primero de ellos lo denominamos apariencia física constó de 12 items que explicaban el 26,5\% de la varianza. En la escala original, este factor se correspondería con condición física. Hemos preferido asignarle otra deno- minación puesto que el contenido de los ítems habla más de la apariencia general de la persona (silueta, estómago, salud, orejas, etc.,) que de la condición física, que parece referirse a un aspecto más específico. Por otra parte, se encontró que uno de los ítems (Piernas), que no formaba parte de la estructura original para hombres, sí que cargó en la nuestra.

El segundo factor lo denominamos atractivo sexual, constó de 13 items que explicaban el $10,79 \%$ de la varianza y también tiene un nombre diferente al de la escala original, porque los items que lo componen reflejan aspectos muy importantes de la sexualidad (actividades sexuales, genitales, impulso sexual), así

Tabla 3. Resultados del análisis factorial en el grupo de varones

\begin{tabular}{|c|c|c|c|}
\hline \multirow[t]{2}{*}{ Item del BES } & \multicolumn{3}{|c|}{ Cargas factoriales } \\
\hline & Apariencia física & Atractivo sexual & Fuerza muscular \\
\hline 24. piernas & 0,874 & & \\
\hline 16. culo / nalgas & 0,848 & & \\
\hline 10. muslos & 0,786 & & \\
\hline 25. silueta & 0,745 & & \\
\hline 35. peso & 0,719 & & \\
\hline 8. cintura & 0,665 & & \\
\hline 23. caderas & 0,607 & & \\
\hline 14. constitución & 0,545 & & \\
\hline 29. estómago & 0,522 & 0,441 & \\
\hline 30. salud & 0,499 & & \\
\hline 11. orejas & 0,490 & & \\
\hline 33. condición física & 0,444 & & 0,455 \\
\hline 17. agilidad & & 0,616 & \\
\hline 22. mejillas & & 0,615 & \\
\hline 34. cara & & 0,590 & \\
\hline 31. actividades sexuales & & 0,590 & \\
\hline 28. genitales & & 0,578 & \\
\hline 15. coordinación física & & 0,569 & \\
\hline 6. labios & & 0,540 & \\
\hline 21. aspecto de los ojos & & 0,512 & \\
\hline 26. impulso sexual & & 0,504 & \\
\hline 2. apetito & & 0,503 & \\
\hline 5. reflejos & & 0,477 & \\
\hline 9. nivel de energía & & 0,442 & \\
\hline 7. fuerza muscular & & & 0,794 \\
\hline 18. anchura de espaldas & & & 0,768 \\
\hline 12. bíceps & & & 0,741 \\
\hline 19. brazos & & & 0,721 \\
\hline 32. vello corporal & & & 0,501 \\
\hline
\end{tabular}

Nota. Análisis de componentes principales, rotación varimax. 
como otras partes del cuerpo que, de alguna manera, podrían reflejar el atractivo sexual de los hombres, como son el aspecto del estómago, la cara, los labios, los ojos, etc.

Finalmente, el último factor se denominó fuerza muscular y contó con 6 items que explicaban el $10,36 \%$ de la varianza. En este caso, sí se mantuvo el nombre original pues, aunque cuenta con menor número de ítems, la mayoría son los mismos que en la estructura original. No obstante, en nuestro caso, se añade un ítem (vello corporal) que en el original no estaba incluido.

\section{Consistencia interna}

Para analizar la consistencia interna, se evaluaron las respuestas de toda la muestra a través del cálculo del coeficiente a de Cronbach tanto para la escala total, como para cada una de las subescalas. En el caso de las mujeres, para la escala total, los resultados mostraron un coeficiente a de 0,906. El rango de correlaciones de los ítems con el total osciló entre 0,10 y 0,65, siendo el ítem que menor correlación presentó el «aspecto de los ojos» y el que mayor correlacionó la "constitución, complexión". Con respecto a cada una de las subescalas, el índice a para la subescala de "atractivo sexual» fue de 0,762 ; oscilando el rango de correlaciones entre 0,35 y 0,56 . El ítem que menor correlación presentó fue "genitales» y el que mayor correlación presentó fue «mejillas / pómulos». Para la subescala de «condición física» el índice a fue de 0,848. Las correlaciones oscilaron entre 0,34 y 0,68 , siendo el ítem de menor correlación el «impulso sexual» y el de mayor correlación el de «condición física». Finalmente, la consistencia interna para la subescala "preocupación por el peso" fue de 0,903. El rango de correlaciones entre los ítems fue de 0,45 a 0,84 , correspondiendo la menor correlación al ítem "apetito» y la mayor al ítem "silueta».

Las correlaciones corregidas (correlaciones del ítem con el total, eliminando el propio ítem) de cada uno de los ítems con el total de la escala fueron superiores a 0,30, excepto para los siguientes ítems: Olor corporal $(-0,043)$, Nariz $(0,11)$, Aspecto de los ojos $(0,10)$, Actividades sexuales $(0,29)$ y Vello corporal $(0,14)$. Por último, señalar que la correlación entre las tres subescalas fue moderada; concretamente la correlación menor se encontró entre las subescalas «atractivo sexual» y "preocupación por el peso» ( $\mathrm{r}$ $=0,42)$. La más alta se encontró entre "condición física» $\mathrm{y}$ "atractivo sexual» $(r=0,56)$. Finalmente, la correlación entre "preocupación por el peso» y «condición física» fue $\mathrm{r}=\mathbf{0 , 5 3 1}$.

En el caso de los varones, para la escala total se obtuvo un coeficiente a de 0,896 . El rango de correlaciones de los ítems con el total osciló entre 0,13 y 0,66, siendo el ítem que menor correlación presentó el "vello corporal» y el que mayor correlacionó el de «silueta». Con respecto a cada una de las subescalas, el índice a para la subescala de "apariencia física» fue de 0,887; oscilando el rango de correlaciones entre 0,42 y 0,76 . El ítem que menor correlación presentó fue "salud» y el que mayor correlación presentó fue el de «piernas». Para la subescala de «atractivo sexual» el índice a fue de 0,828 . Las correlaciones oscilaron entre 0,29 y 0,54 , siendo el ítem de menor correlación el item «reflejos» y el de mayor correlación el ítem "cara». Finalmente, la consistencia interna para la subescala «fuerza muscular» fue de 0,809. El rango de correlaciones entre los ítems fue de 0,30 a 0,72 , correspondiendo la menor correlación al ítem de "vello corporal» y la mayor al ítem de "anchura de espalda».

Las correlaciones corregidas (correlaciones del ítem con el total, eliminando el propio ítem de cada uno de los ítems con 
el total de la escala fueron superiores a 0,30 , excepto para los siguientes ítems: Olor corporal $(0,13)$, Apetito $(0,17)$; Nariz $(0,11)$, Reflejos $(0,29)$; Bíceps $(0,26)$; Pies $(0,20)$ y Vello corporal $(0,13)$. Por último, señalar que la correlación entre las tres subescalas fue más baja que en la población femenina. Concretamente la correlación menor se encontró entre las subescalas «atractivo sexual» $y$ «fuerza muscular» $(r=0,32)$; la más alta se encontró entre «apariencia física» $y$ «atractivo sexual» $(r=0,46) y$, finalmente, la correlación entre «fuerza muscular» $\mathrm{y}$ «apariencia física» fue de $\mathrm{r}=\mathbf{0 , 3 8}$.

\section{Fiabilidad test-retest}

Con objeto de analizar la fiabilidad test-retest o estabilidad temporal (un mes) se calculó el coeficiente de correlación interclases. Se calculó la fiabilidad para el total de la escala y para las subes- calas de la estructura femenina, por un lado, y la estructura masculina, por otro. Los resultados indicaron que existe una alta fiabilidad entre las medidas del prey las del retest: $0,87(0,91-0,82)$ pasado un mes para la escala total. En cuanto a la estabilidad temporal de las subescalas es moderada tanto en el caso de las mujeres 0,67 $(0,59-0,74)$, como de los varones $0,64(0,46-0,77)$.

Diferencias de grupo en las subescalas del BES

Las medias y desviaciones típicas de los diferentes grupos se ofrecen en la Tabla 4 para mujeres y en la Tabla 5 para varones.

(1) Diferencias en función del género. En primer lugar, se aplicó un ANOVA con un factor entre-grupos (género), teniendo como variable dependiente la puntuación

Tabla 4. Medias y desviaciones típicas para el grupo de mujeres

\begin{tabular}{|c|c|c|c|c|c|c|c|c|c|c|c|c|}
\hline & \multicolumn{3}{|c|}{ Atractivo sexual } & \multicolumn{3}{|c|}{$\begin{array}{l}\text { Preocupación } \\
\text { por el peso }\end{array}$} & \multicolumn{3}{|c|}{ Condición física } & \multicolumn{3}{|c|}{ BES total } \\
\hline & $\mathbf{N}$ & Media & DT & $N$ & Media & DT & $\mathrm{N}$ & Media & DT & $\mathrm{N}$ & Media & DT \\
\hline \multicolumn{13}{|l|}{ Grupos de edad } \\
\hline $14-16$ & 101 & 27,50 & 5,02 & 91 & 26,40 & 8,55 & 92 & 35,40 & 7,15 & 110 & 94,28 & 19,20 \\
\hline $17-22$ & 61 & 28,50 & 4,25 & 65 & 28,52 & 8,63 & 62 & 38,03 & 6,27 & 67 & 101,83 & 16,90 \\
\hline $23-29$ & 34 & 28,82 & 3,80 & 33 & 30,27 & 6,58 & 34 & 39,80 & 6,60 & 34 & 105,76 & 15,17 \\
\hline \multicolumn{13}{|l|}{ Grupos de IMC } \\
\hline$<19$ & 47 & 28,02 & 4,50 & 47 & 30,82 & 7,31 & 45 & 38,02 & 6,34 & 54 & 101,33 & 17,77 \\
\hline $19-20$ & 60 & 28,46 & 4,47 & 57 & 30,22 & 7,53 & 60 & 37,75 & 6,85 & 62 & 102,38 & 19,38 \\
\hline $21-23$ & 67 & 27,90 & 4,25 & 65 & 24,73 & 7,84 & 60 & 36,93 & 7,12 & 71 & 94,60 & 18,37 \\
\hline$\geq 24$ & 21 & 27,90 & 6,30 & 20 & 23,75 & 9,90 & 22 & 35,95 & 7,79 & 23 & 92,78 & 18,76 \\
\hline \multicolumn{13}{|c|}{ Grupos de Esquematismo } \\
\hline No esquemático & 88 & 28,26 & 4,11 & 86 & 29,32 & 6,31 & 86 & 37,63 & 6,31 & 95 & 101,51 & 14,25 \\
\hline Esquemático gordo & 32 & 26,12 & 5,36 & 33 & 19,51 & 9,20 & 25 & 33,36 & 8,36 & 34 & 82,97 & 20,94 \\
\hline Esquemático justo & 42 & 28,80 & 5,19 & 40 & 28,40 & 7,28 & 43 & 38,25 & 6,91 & 47 & 100,27 & 18.25 \\
\hline Esquemático delgado & 21 & 29,38 & 4,30 & 19 & 35 & 6,29 & 21 & 40,90 & 5,53 & 22 & 109,22 & 23,92 \\
\hline \multicolumn{13}{|l|}{ Grupos EAT-40 } \\
\hline Población normal & 149 & 28,35 & 4,18 & 141 & 29,33 & 7,04 & 144 & 37,61 & 6,40 & 158 & 101,31 & 16,78 \\
\hline Población subclínica & 43 & 27,39 & 5,61 & 44 & 23,06 & 10,22 & 42 & 36,47 & 8,23 & 49 & 90,44 & 21,97 \\
\hline \multicolumn{13}{|l|}{ Grupos RS } \\
\hline Restrictivos & 36 & 26,94 & 5,64 & 36 & 21,55 & 9,35 & 32 & 35,62 & 7,50 & 39 & 87,48 & 20,57 \\
\hline No restrictivos & 149 & 28,31 & 4,10 & 144 & 29,38 & 7,40 & 149 & 37,71 & 6,76 & 161 & 101,27 & 17,39 \\
\hline
\end{tabular}


Tabla 5. Medias y desviaciones típicas para el grupo de varones

\begin{tabular}{|c|c|c|c|c|c|c|c|c|c|c|c|c|}
\hline & \multicolumn{3}{|c|}{ Apariencia física } & \multicolumn{3}{|c|}{ Atractivo sexual } & \multicolumn{3}{|c|}{ Fuerza muscular } & \multicolumn{3}{|c|}{ BES total } \\
\hline & $\mathrm{N}$ & Media & DT & $\mathrm{N}$ & Media & DT & $N$ & Media & DT & $\mathrm{N}$ & Media & DT \\
\hline \multicolumn{13}{|l|}{ Grupos de edad } \\
\hline $14-17$ & 39 & 39,77 & 6,82 & 37 & 48,65 & 6,20 & 40 & 22,05 & 3,71 & 43 & 109,72 & 10,95 \\
\hline $18-27$ & 26 & 42,73 & 7,77 & 28 & 49,82 & 7,02 & 29 & 20,76 & 4,64 & 28 & 114,53 & 15,18 \\
\hline \multicolumn{13}{|l|}{ Grupos de IMC } \\
\hline $19-21$ & 39 & 41,84 & 7,08 & 40 & 49,55 & 6,88 & 41 & 20,43 & 4,16 & 43 & 111,65 & 13,38 \\
\hline$\geq 22$ & 25 & 39,76 & 7,69 & 24 & 48,79 & 6,01 & 27 & 23,14 & 3,70 & 27 & 111,92 & 12,94 \\
\hline \multicolumn{13}{|l|}{ Grupos Esquematismo } \\
\hline No esq & 41 & 39,93 & 7,71 & 40 & 47,55 & 6,48 & 42 & 21 & 4,44 & 43 & 109,69 & 12,49 \\
\hline Esquemé & 2 & 40,50 & 12,02 & 2 & 46,50 & 7,77 & 3 & 22 & 3,00 & 3 & 111 & 17,57 \\
\hline Esquem & 7 & 44,42 & 6,97 & 8 & 51,37 & 5,60 & 8 & 21,62 & 3,20 & 8 & 114,75 & 12,89 \\
\hline Esquemáti & 8 & 42,62 & 7,34 & 8 & 55,12 & 5,11 & 8 & 21,87 & 5,08 & 8 & 119,75 & 12,76 \\
\hline \multicolumn{13}{|l|}{ Grupos EAT-4O } \\
\hline Població & 55 & 40,76 & 6,27 & 54 & 47,83 & 5,78 & 57 & 21,15 & 3,90 & 59 & 110,88 & 13,07 \\
\hline Población subclínica & 4 & 36,50 & 15,67 & 4 & 55,75 & 7,08 & 4 & 19,25 & 5,67 & 4 & 111,75 & 16,78 \\
\hline \multicolumn{13}{|l|}{ Grupos RS } \\
\hline Restri & 4 & 42,50 & 4,79 & 4 & 48,25 & 4,35 & 4 & 22,25 & $\mathbf{3}$ & 4 & 113 & 8,40 \\
\hline No restrictivos & 49 & 40,22 & 7,55 & 48 & 48,50 & 6,58 & 51 & 20,98 & 4,19 & 52 & 111,42 & 13,52 \\
\hline
\end{tabular}

total en la BES. Los resultados indicaron diferencias significativas $(F(1,281)=$ 29,744, $\mathrm{p}<, 005$ ), en el sentido de que los varones obtenían puntuaciones más elevadas en la estima corporal que las mujeres.

Posteriormente, se aplicaron diversos ANOVAs considerando, por una parte, las subescalas de la estructura para las mujeres y, por otra, las de los varones. En el caso de las subescalas femeninas, se obtuvieron nuevamente diferencias significativas en las tres subescalas, en el sentido de que los varones obtenían puntuaciones más elevadas: "Atractivo físico» $(F(1,282)=8,540, p<, 005)$; «Preocupación por el peso» $(F(1,281)=27,546$, $\mathrm{p}<, 005)$; «Condición física» $(\mathrm{F}(\mathbf{1 , 2 8 1 )}=$ $21,499, p<, 005)$. Por último, en las subescalas masculinas, se encontraron también diferencias significativas en la misma dirección: "Apariencia física» $(F(1,261)$ $=21,097, \mathrm{p}<, 005)$; "Atractivo sexual" $(F(1,236)=21,584, p<, 005)$; "Fuerza muscular» $(\mathrm{F}(1,269)=33,929, \mathrm{p}<, 005)$.

(2) Diferencias en función de la edad. Con el fin de comprobar si existían dife- rencias en cuanto a la estima corporal en función de la edad, se establecieron diferentes grupos de edad para las mujeres (de 14 a 16; de 17 a 22; y de 23 a 29) y para los varones (de 14 a 17 y de 18 a 27), debido a la diferente distribución de ambos grupos en cuanto a su tamaño. Se hicieron análisis separados para hombres y para mujeres, empleando en los ANOVAS como factor entre-grupos la edad y tomando como variables dependientes las puntuaciones de la muestra en cada una de las subescalas del BES, así como en el BES total. En el caso de las mujeres (ver Tabla 4), los resultados indicaron que las chicas más jóvenes (de 14 a 16 años) eran las que menos satisfechas se encontraban con su cuerpo cuando se tenía en cuenta la puntuación total de la escala $(F(2,208)=6,684, p<, 005)$, y también las que menos satisfechas estaban con su peso $(F(2,186)=3,032, p<, 005), y$ con su condición física: $(\mathrm{F}(2,185)=$ $4,383, p<, 005$ ). No hubo diferencias en la subescala de «atractivo físico». En el caso de los varones (ver Tabla 5), los resulta- 
dos no mostraron diferencias significativas en función de la edad ni en la puntuación total, ni en las tres subescalas.

(3) Diferencias en función del IMC. Para analizar las diferencias en la estima corporal en función de la IMC, los análisis se aplicaron por separado a mujeres y a varones. En ambos casos los ANOVAs empleaban como variables dependientes las puntuaciones de la muestra en cada una de las subescalas del BES, así como en el BES total. Se tomaba como factor entre-grupos el IMC. Para dividir estos grupos, y debido al tamaño y distribución de las muestras, no se tuvo en cuenta la tabla propuesta por la OMS (Organización Mundial de la Salud: < 16 «peso extremadamente bajo»; 16-18 «significativamente bajo»; 20-25 "peso saludable»; 27-30 "sobrepeso»; 30-40 «obesidad»; $>40$ «obesidad mórbida»). En el caso de las mujeres de nuestra muestra, la mayor parte (el $55.23 \%$ ) contaba con un IMC que oscilaba entre 18 y 21 , y en el caso de los varones la mayoría $(52,11 \%)$ se encontraba con un IMC entre 19 y 21 ; de tal manera que el establecer grupos adecuándonos a los rangos propuestos por la OMS, daba como resultado grupos muy dispares en cuanto a su tamaño. Debido a este problema, se decidió establecer cuatro grupos de IMC para mujeres (IMC $<19$, IMC entre 19-20; IMC entre 21-23; IMC $\geq 24$ ) y dos grupos para los varones (IMC entre 19-21; IMC $\geq 22$ ), puesto que el tamaño de la muestra masculina era menor y la forma que en que se distribuían en función del IMC también era diferente. Los resultados indicaron que, para la muestra femenina (ver Tabla 4), existían diferencias significativas entre los grupos de IMC en la puntuación total de la escala, encontrando que las participantes que mostraban menor IMC parecían encontrarse más satisfechas, de manera global, con su cuerpo $(F(3,206)=3,099$, $\mathrm{p}<, 005$ ). En cuanto a las subescalas, sólo se encontraron diferencias significativas para "preocupación por el peso", siendo de nuevo las participantes de menor IMC las que más satisfechas se encontraban con su peso $(F(3,206)=9,182, p<, 005)$. Para el caso de los varones (ver Tabla 5), los resultados mostraron diferencias significativas entre los grupos de IMC sólo en la subescala "fuerza muscular", siendo los participantes con mayor IMC los que más satisfechos se encontraban con este aspecto del cuerpo $(F(1,66)=7,514$, $\mathrm{p}<, 005$ ).

(4) Diferencias en función del esquematismo. De acuerdo con lo indicado más arriba, la variable esquematismo diferenciaba 4 grupos (no esquemático, esquemático justo, esquemático gordo y esquemático delgado). También se analizaron los resultados por separado para varones y para mujeres, empleando en los ANOVAs como variables dependientes las puntuaciones de la muestra en cada subescala del BES, y en el BES total. Para las mujeres (ver Tabla 4), los resultados indicaron que las chicas clasificadas como "esquemática delgadas», esto es, aquellas que se auto-calificaban como delgadas y esto era una característica importante en su definición de sí mismas, eran las que más satisfechas se encontraban con su cuerpo, si tenemos en cuenta la puntuación total de la escala $(\mathrm{F}(3,194)=12,432, \mathrm{p}<, 005)$, y también las que más satisfechas están con su peso $(\mathrm{F}(3,194)=22,620, \mathrm{p}<, 005)$ y con su condición física $(F(3,194)=5,170, p<0,005)$. No se encontraron diferencias para el atractivo físico. En el caso de los varones (ver Tabla 5), los resultados indicaron que los chicos clasificados como "esquemático delgados» eran los que más a gusto se encontraban con las partes del cuerpo relacionadas con el atractivo sexual $(F(4,54)=2,839, p<0,005)$. No se encontraron diferencias ni en la puntuación total ni en las otras dos subescalas.

(5) Diferencias en función de la puntuación en el EAT. Como se ha comenta- 
do, el EAT-40 permite diferenciar entre población general y subclínica, siendo el punto de corte 20 . También aquí los datos se analizaron por separado en función del género. Los resultados obtenidos en el ANOVA para la muestra femenina (ver Tabla 4) indicaron que las chicas con puntuaciones menores de 20 en el EAT-40 se encontraban más satisfechas con su cuerpo, teniendo en cuenta la puntuación total $(F(1,205)=13,417, p<0,005)$ y también estaban más satisfechas con su peso $(F(1,205)=21,045, p<0,005)$. Para los varones (ver Tabla 5), los resultados indicaron que aquellos con una puntuación mayor de 20 en el EAT-40, se encontraban más satisfechos con su atractivo sexual $(F(1,56)=6,682, p<0,005)$. No hubo diferencias en el resto de las variables.

(6) Diferencias en función de la puntuación en la Escala de Restricción. Finalmente, se tuvo en cuenta la puntuación en la «Escala de Restricción» (RS), la cual permite clasificar a los participantes en "restrictivos» en cuanto a su patrón alimentario, cuando la puntuación total obtenida es igual o superior a 16, y en "no restrictivos», cuando la puntuación es menor a 16. Los resultados indicaron, en el caso de las mujeres que las chicas «restrictivas» estaban menos satisfechas con su cuerpo, en la puntuación total $(F(1,198)=18,329$, $\mathrm{p}<0,005)$, y más preocupadas por su peso $(F(1,198)=28,784, p<0,005)$. En este caso, para los varones, no se obtuvieron resultados de significación estadística.

\section{Validez convergente}

Con el fin de analizar las relaciones existentes entre la puntuación total del BES y la de sus subescalas (tanto de la estructura de mujeres, como de la de los varones) con otras medidas empleadas en la evaluación de la IC, se calcularon coeficientes de correlación momento producto de Pearson por separado para mujeres (ver Tabla 6) y varones (ver Tabla 7).

Para la muestra femenina, los resultados indicaron que la puntuación total del BES correlacionaba significativamente con todas las medidas de IC empleadas. En cuanto a las subescalas del BES, tanto la "preocupación por el peso" como «condición física» mostraron correlaciones con todas las demás medidas de comparación; sin embargo, la subescala «atractivo sexual», sólo mostró correlaciones con cinco medidas, cuatro de ima-

Tabla 6. Correlaciones para el grupo de mujeres

\begin{tabular}{lcccc}
\hline & Atractivo Sexual & $\begin{array}{c}\text { Preocupacion } \\
\text { por el peso }\end{array}$ & Condición Física & BES total \\
\hline BAT & $-0,230^{\star \star}$ & $-0,642^{\star \star}$ & $-0,452^{\star \star}$ & $-0,588^{\star \star}$ \\
BIATQ & $-0,301^{\star \star}$ & $-0,555^{\star \star}$ & $-0,446^{\star \star}$ & $-0,579^{\star \star}$ \\
BASS & $0,386^{\star \star}$ & $0,646^{\star \star}$ & $0,475^{\star \star}$ & $0,623^{\star \star}$ \\
SIBID & $-0,185^{\star \star}$ & $-0,541^{\star \star}$ & $-0,369^{\star \star}$ & $-0,490^{\star \star}$ \\
BSQ & $-0,159$ & $-0,533^{\star \star}$ & $-0,372^{\star \star}$ & $-0,474^{\star \star}$ \\
ASI & $-0,147$ & $-0,411^{\star \star}$ & $-0,293^{\star \star}$ & $-0,390^{\star \star}$ \\
BIAQ & $-0,114$ & $-0,433^{\star \star}$ & $-0,315^{\star \star}$ & $-0,409^{\star \star}$ \\
EAT-40 & $-0,097$ & $-0,347^{\star \star}$ & $-0,176^{*}$ & $-0,273^{\star \star}$ \\
RS & $-0,110$ & $-0,534^{\star \star}$ & $-0,308^{\star \star}$ & $-0,434^{\star \star}$ \\
RSES & $0,224^{\star \star}$ & $0,423^{\star \star}$ & $0,437^{\star \star}$ & $0,459^{\star \star}$ \\
\hline
\end{tabular}

BAT: Test de las Actitudes Corporales; BIATQ: Cuestionario sobre los Pensamientos Automáticos acerca de la Imagen Corporal; BASS: Escala de Satisfacción de Zonas Corporales; SIBID; Inventario de malestar provocado por las situaciones relacionadas con la imagen corporal; BSQ: Cuestionario de la Forma Corporal; ASI: Inventario sobre los Esquemas acerca de la Apariencia; BIAQ: Cuestionario sobre la Evitación de la Imagen Corporal; EAT-40 Cuestionario de las actitudes hacia la comida; RS: Escala de Restricción; RSES: Cuestionario de Autoestima de Rosenberg, ${ }^{\star *} p<0,01 ;{ }^{\star} p<0,05$ (Bilateral). 
Tabla 7. Correlaciones para el grupo de varones

\begin{tabular}{|c|c|c|c|c|}
\hline Otras medidas de TA & Apariencia física & Atractivo sexual & Fuerza muscular & BES total \\
\hline BAT & $-0,351 * *$ & $-0,027$ & 0,068 & $-0,175$ \\
\hline BIATQ & $-0,199$ & 0,007 & $-0,216$ & $-0,156$ \\
\hline BASS & $0,334^{\star *}$ & 0,225 & 0,074 & $0,412 * \star$ \\
\hline SIBID & $-0,057$ & 0,148 & $-0,021$ & $-0,070$ \\
\hline BSQ & $-0,193$ & 0,058 & 0,137 & $-0,186$ \\
\hline ASI & 0,168 & 0,194 & 0,010 & 0,105 \\
\hline BIAQ & $-0,070$ & 0,175 & $-0,033$ & $-0,110$ \\
\hline EAT-40 & 0,055 & $0,354^{* *}$ & 0,214 & 0,184 \\
\hline RS & $-0,025$ & 0,037 & 0,110 & $-0,100$ \\
\hline RSES & 0,123 & 0,196 & 0,112 & 0,174 \\
\hline
\end{tabular}

BAT: Test de las Actitudes Corporales; BIATQ: Cuestionario sobre los Pensamientos Automáticos acerca de la Imagen Corporal; BASS: Escala de Satisfacción de Zonas Corporales; SIBID; Inventario de malestar provocado por las situaciones relacionadas con la imagen corporal; BSQ: Cuestionario de la Forma Corporal; ASI: Inventario sobre los Esquemas acerca de la Apariencia; BIAQ: Cuestionario sobre la Evitación de la Imagen Corporal; EAT-40 Cuestionario de las actitudes hacia la comida; RS: Escala de Restricción; RSES: Cuestionario de Autoestima de Rosenberg.

${ }^{\star *} p<0,01 ;{ }^{*} p<0,05$ (Bilateral).

gen corporal ( «Test de Actitudes Corporales» -BAT-, «Cuestionario sobre los Pensamientos Automáticos acerca de la imagen Corporal» -BIATQ-, «Inventario de Malestar Provocado por las Situaciones Relacionadas con la Imagen Corporal» - SIBID_, : y «Escala de Satisfacción de Zonas Corporales»-BASS-), y una de auto-estima general (Autoestima de Rosenberg -RSES).

Por lo que se refiere a la muestra de varones, los resultados indicaron que la puntuación total del BES únicamente correlacionó con la «Escala de Satisfacción de Zonas Corporales» (BASS). En cuanto a las subescalas, «apariencia física» sólo mostró correlación con el «Cuestionario de actitudes hacia el propio cuerpo» (BAT) y con la Escala de Satisfacción de Zonas Corporales (BASS); la subescala «atractivo sexual» únicamente correlacionó con el EAT-40, y finalmente, la subescala «fuerza muscular» no correlacionó con ninguno de los instrumentos utilizados.

\section{DISCUSIÓN}

De acuerdo con los resultados obtenidos, se puede afirmar que la adaptación reali- zada de la escala BES posee garantías psicométricas suficientes para se aplicada en la población española. La escala ha mostrado tener muy buena consistencia interna, tanto en su totalidad, como en cada una de las subescalas, y tanto en población femenina como masculina. Además, la escala parece tener una buena estabilidad temporal, ya que los datos indican que, transcurrido un mes, las puntuaciones obtenidas en el primer pase se mantuvieron similares en el segundo.

Por lo que se refiere a la estructura factorial, en el caso de la población femenina, se obtuvieron, al igual en el estudio original, tres factores, denominados de la misma manera (Preocupación por el peso, Condición física y Atractivo sexual). No obstante, hubo alguna algunas diferencias en cuanto a la composición de estos factores. Así, en primer lugar, nuestra estructura cuenta con 4 ítems menos (olor corporal, nariz, pechos, y vello corporal), todos incluidos en el factor «atractivo sexual» de Franzoi y Shields, pero que en nuestro estudio no cargaron en ningún factor. Quizá esto se deba a diferencias en las muestras empleadas: la mayor parte de la de este estudio era más joven (rango de edad de 14 a 29) 
que la original (todas mujeres universitarias), contando además con que el mayor número de participantes estaban agrupadas dentro del rango de 14 a 17 años (72 participantes $-63 \%$ de la muestra). En este sentido, se podría argumentar que a esas edades, a las adolescentes no les preocupa tanto o les son menos relevantes aspectos tan concretos relacionados con el atractivo sexual (como el olor o el vello corporal, los pechos, etc), aspectos que, sin embargo, pudieran ser más importantes o relevantes a edades superiores. Como ya se ha señalado, los propios autores de la escala reconocían que la muestra incluida en su desarrollo no representaba a todos los grupos de edad y que sería necesario contar con normas descriptivas adecuadas para cada grupo de edad (Franzoi y Shields 1984).

También con relación al factor de «atractivo sexual» hemos de señalar otras diferencias con la escala original. Concretamente en nuestro estudio no se incluye el ítem «impulso sexual» pero sí el de "salud». En el primer caso, este ítem (impulso sexual) aparece como parte del factor «Condición física». Es decir, parece que para la población española femenina la satisfacción con el impulso sexual podría implicar más una acción relacionada con la condición física general de la persona, que un rasgo que pueda guardar relación con el atractivo sexual. En cuanto al segundo ítem (salud), en la escala original forma parte del factor «condición física», pero en nuestra estructura aparece tanto en el factor de "condición física» como en el de «atractivo sexual», indicando nuevamente una relación entre satisfacción con la salud y satisfacción con otros aspectos del atractivo sexual.

Por lo que respecta a los otros dos factores, el referido a «preocupación por el peso" es idéntico al original, tanto en el número de ítems que lo componen (10), como en el contenido de los mismos. Por último, en la «condición física», a dife- rencia del original, se incluyen dos ítems más (actividades sexuales e impulso sexual). Esta diferencia podría tener relación con señalado anteriormente, es decir, quizás, para la población española femenina la satisfacción con el impulso sexual y las actividades sexuales se relacionan más con la condición física general de la persona, que con el atractivo físico.

Por lo que respecta a la estructura obtenida para los varones, difiere de la encontrada en el estudio original. En primer lugar, nuestra estructura cuenta con 5 ítems menos que el original. Por otro lado, aunque la estructura factorial también consta de tres factores, sin embargo estos son diferentes tanto en número de ítems como en el contenido de los mismos. Estos factores fueron: «Apariencia física», "Atractivo sexual" y»Fuerza muscular». Quizás pueda deberse a que en nuestro trabajo el número de participantes fue menor $(\mathrm{N}=72)$ que en el estudio de Franzoi y Shields $(\mathrm{N}=331)$. Además, el rango de edad de nuestros participantes fue más heterogéneo (de 14 a 27 años, de los cuales el mayor número de participantes se concentró entre los 14 y 18 años), que el empleado por los otros autores (muestra compuesta sólo por estudiantes universitarios). Asimismo, también podrían existir diferencias culturales entre la muestra española y la americana

Por otra parte, los resultados obtenidos indican que la estima corporal, en la población española, parece estar influenciada por variables como el género, la edad, el IMC, pero también por otras características como el nivel de «esquematismo" corporal, la restricción alimentaria o la presencia / ausencia de características típicas de la anorexia nerviosa.

En cuanto al género, nuestros datos indican que a las mujeres su cuerpo les suscita menos sentimientos positivos que a los varones. Además, también se obser- 
va un perfil diferente de estima corporal en varones y en mujeres. Así, los datos apuntan a que las mujeres que se perciben delgadas, y eso les importa (clasificadas como "esquemáticas delgadas») están más satisfechas con su cuerpo. En una línea similar, las chicas que tiene un IMC más bajo parecen tener sentimientos más positivos hacia su cuerpo, y preocuparse menos por su peso. Esto resulta de gran importancia si tenemos en cuenta las características de los trastornos alimentarios. En el caso de las personas que sufren estos trastornos, aún a pesar de estar delgadas en exceso, se sienten poco satisfechas con su cuerpo; sin embargo, en la población general, no patológica, la delgadez es sinónimo de satisfacción corporal.

Por otro lado, este estudio también indica que los grupos que podrían calificarse como de "riesgo» a padecer un trastorno alimentario se caracterizan por tener una menor estima corporal. De hecho, en este estudio, las participantes que obtuvieron una puntuación en el EAT-40 mayor de 20 (subclínicas) se encontraban menos satisfechas a nivel global con su cuerpo y les preocupaba más su peso (como ocurre en la población clínica). Por otra parte, también se observa que las chicas más jóvenes (entre 14 y 16 años) y las que fueron clasificadas como "restrictivas" en cuanto a su patrón alimentario (según la puntuación obtenida en la «Escala de Restricción»), se encontraban menos satisfechas con su cuerpo.

Sin embargo, en el caso de los varones los resultados eran diferentes. En primer lugar, llama la atención el que existan menos diferencias entre grupos en el caso de los varones. Así, y a diferencia de las mujeres, la estima corporal de los varones no parece varia con la edad, ni con el grado de restricción alimentaria. En segundo lugar, los datos indican que los chicos con mayor IMC eran los que se encontraban más satisfechos con su cuer- po, concretamente con las partes que guardaban relación con la fuerza muscular. Este dato estaría señalando el papel diferencial que desempeña el IMC en la estima corporal de los hombres y las mujeres: mientras que las chicas prefieren estar delgadas, y están más satisfechas cuando lo consiguen, los varones con una mayor complexión corporal se encuentran más satisfechos de su cuerpo y, más en concreto, con los aspectos de éste que reflejan su fuerza muscular. Este dato podría estar relacionado con la mayor incidencia de problemas de anorexia restrictiva en mujeres y la mayor incidencia de problemas de vigorexia en varones. Todo esto parece reflejar la influencia de los cánones culturales en nuestra sociedad, es decir, cómo los valores estéticos acerca de la delgadez están dejando mella, en mayor medida, en las mujeres, así como la satisfacción que éstas sienten con su cuerpo al aproximarse a esos cánones de belleza, convirtiéndose, de esta manera, en población de riesgo de trastorno alimentario.

Por lo que se refiere a la validez convergente de la escala, hay que indicar que tanto la escala en su totalidad (puntuación total), como las subescalas "preocupación por el peso« y «condición física» correlacionan significativamente con el resto de medidas de IC, con una medida autoestima (RSES), con una medida de restricción alimentaria (RS) y con una medida de sintomatología de trastornos alimentarios (EAT-40). La subescala de «atractivo sexual» es la que menos correlaciones muestra con otras medidas de IC. No ocurrió lo mismo con la muestra de varones en la que, ni la escala en su totalidad, ni sus subescalas mostraron tener esa adecuada validez convergente, que sí observamos en el caso de las mujeres. Parece por tanto que, en el caso de los varones, el componente afectivo de la IC, o más concretamente la estima corporal, no muestra claras relaciones con 
otros componentes cognitivos y conductuales. Sin embargo, este dato hay que tomarlo con precaución, ya que la muestra masculina adolecía de un $\mathrm{N}$ significativamente menor.

Así pues, podríamos concluir diciendo que la Escala de Estima Corporal parece ser un instrumento adecuado para la evaluación de la imagen corporal, fundamentalmente en mujeres españolas; asimismo y, como se ha observado en numerosos estudios, nosotros también hemos podido comprobar que el concepto de estima corporal es un constructo multidimensional y que se puede ver afectado por la influencia de diferentes variables (sexo, edad, IMC, esquematismo, restricción alimentaria).

Por último, hemos de señalar que nuestro estudio cuenta con algunas limitaciones que sería recomendable subsanar en investigaciones posteriores, siendo la fundamental el limitado tamaño de la muestra de varones. Consideramos que si hubiéramos podido contar con un mayor número de varones, los resultados obtenidos hubieran sido más satisfactorios.

\section{BIBLIOGRAFÍA}

Baños, R. M. y Guillén, 5. (2000). Psychometric characteristics in normal and social phobic samples for a Spanish version of de Rosenberg Self-Esteem Scale. Psychological Reports, 87, 269-274.

Carter, P. I. y Moss, R. A.(1984). Screening for anorexia and bulimia nervosa in a college population: Problems and limitations. Addictive Behaviors, 9(4),417-419.

Cash, T. F. (1990). The psychology of physical appearance: Aesthetics, attributes, and images. In T. F. Cash y T. Pruzinsky (Eds.), Body images: Development, deviance, and change (pp.51-79). New York: Guilford Press.

Cash, T.F. (1991). Body image therapy: a program for self-directed change. New York: Guilford Press.
Cash, T. F. (1994). The situational inventory of Body-Image Dysphoria: Contextual assessment of a negative body image. The Behavior Therapist, 17, 133-134.

Cash, T. F. y Deagle, E. A. (1997). The nature and extent of body image disturbances in anorexia nervosa and bulimia nervosa: A meta-analysis. International Journal of Eating Disorders, 22, 107-125.

Cash, T. F. y Labarge, A. S. (1996). Development of the Appearance Schemas Inventory: a new cognitive body-image assessment. Cognitive Therapy and Research, 20(1), 37-50.

Castro, J., Toro, J., Salamero, M. y Guimerá, E. (1991). The Eating Attitudes Test: Validation of the Spanish version. Psychological Assessment, 7, 175-190.

Celcil H. y Stanley, M.A. (1997). Reliability and validity of adolescents' scores on the Body Esteem Scale. Educational \& Psychological Measurement, 57 (2), 340-356.

Cooper, P. J., Taylor, M. J., Cooper, Z. y Fairburn, C. G. (1986). The development and validation of The Body Shape Questionnaire. International Journal of Eating Disorders, 6, 485-494.

Fernánez, M.C. y Solé, J (2003). Protocolo de las sesiones de terapia interpersonal aplicada a un grupo de familiares de pacientes con trastorno de conducta alimentaria. Revista de Psicopatología y Psicología Clínica, 8, 67-77.

Fisher, S. (1964). Sex differences in body perception. Psychological Monographs, 78, 122.

Franzoi, S.L. (1994). Further evidence of the reliability and validity of the Body Esteem Scale. Journal of Clinical Psychology, 50, 237-239

Franzoi, S. L. y Shields, S. A. (1984). The body esteem scale: Multidimensional structure and sex differences in college population. Journal of Personality Assessment, 48, 173-178.

Garner, D. M. y Garfinkel, P. E. (1979). The Eating Attitudes Test: An index of the symptoms of anorexia nervosa. Psychology and Medicine, 9, 273-279.

Garner, D. M., Olmsted, M. P. y Polivy, J. (1983). Development and validation of a multidimensional Eating Disorder Inven- 
tory for anorexia nervosa and bulimia. International Journal of Eating Disorders, 2, 15-34.

Gila, A., Castro, M.J. 0, Gómez, J., Toro, J.y Salamero, M. (1999). The body attitude test: Validation of the Spanish version" Eating and Weight Disorders, 4, 175- 178

Gunderson, E. K. y Johnson, L. C. (1965). Past experience, self-evaluation, and present adjustment. Journal of Social Psychology, $66,311-321$.

Heatherton, T. F., Herman, C. P., Polivy, J., King, G. A. y McGree, S. T. (1988). The (miss)measurement of restraint and analysis of conceptual and psychometric issues. Journal of abnormal Psychology, 97, 19-28.

Herman, C. P. y Polivy, J. (1975). Anxiety, restraint and eating behavior. Journal of Abnormal Psychology, 84,66-72.

Mendelson, B.K., Mendelson, M.J y. White, D.R. (2001). Body-Esteem Scale for Adolescents and Adults. Journal of Personality assessment, 76(1),90-106.

Perpiñá, C., Botella, C. y Baños, R. M. (2000). Imagen corporal en los trastornos alimentarios. Evaluación y tratamiento mediante realidad virtual. Valencia: Promolibro.

Probst, M., Vanderycken, W., Van Coppenolle, H. y Vanderlinden, J. (1995). Body Attitude Test for patients with an eating disorder: psychometric characteristics of a new questionnaire. Eating Disorders: The Journal of Treatment and Prevention, 3, 133-145.

Raich, R. M. (2000). Imagen corporal: Conocer y valorar el propio cuerpo. Editorial Pirámide.

Raich, R. M., Mora, M., Soler, Avila,C. Clos, I y Zapater, L. (1996). Adaptación de u instrumento de evaluación de la insatisfacción corporal Clínica y Salud, 7(1) 51-66.
Rosen, J. C., Srebrik, D. y Salzberg, E. (1990). Development of a Body Image Avoidance Questionnaire. Journal of Consulting and Clinical Psychology, 3, 32-37.

Rosenberg,, M. (1965). Society and the Adolescent Self-Image. Princeton: Princeton University Press.

Secord, P. F. y Jourard, S. M. (1953). The appraisal of body-cathexis: Body Cathexis and self. Journal of Consulting Psychology, 17,343-347.

Thomas, C. D., \& Freeman, R. J. (1990). The Body Esteem Scale: Construct validity of the female subscales. Journal of Personality Assessment, 54, 204-212.

Thompson, M. A. (1990). Body-image disturbance: Assessment and treatment. Elmsford, NY: Pergamon Press.

Thompson, M. A. (1995). Assessment of Body Image. In Allison (editor). Handbook of assessment methods for eating behaviors and weight-related problems. Measures, theory and research. Sage Publications.

Thompson, M. A. (1996). Assessing Body Image disturbance: measures, methodology, and implementation. En J. K. Thompson (Ed.), Body Image, eating disorders and obesity. Washington, DC.: APA.

Tucker, L. A. (1981). Internal structure, factor satisfaction, and reliability of the Body Cathexis Scale. Perceptual and Motor Skills, 53, 891-896.

Wear, R. 5. y Pratz, O. (1987). Test-retest reliability for the Eating Disorders Inventory. International Journal of Eating Disorders, 6, 767-769.

Wylie, R. C. (1989). Measures of self-concept. Lincoln, EN: Buros Institute of Mental Measurements. 